\title{
THE REGISTRATION AND THE PROCESSING OF SIGNALS OF GEOMAGNETIC PULSATIONS IN THE SYSTEM OF THE GEODYNAMIC MONITORING
}

\author{
O. R. Kuzichkin ${ }^{1 *}$, A. V. Grecheneva ${ }^{1}$, R. P. Gakhov ${ }^{1}$, N. V. Dorofeev ${ }^{2}$, A. A. Bykov ${ }^{2,}$ B. R.
} Gakhov $^{1}$

\author{
${ }^{1}$ Belgorod State University, 85 Pobedy St, Belgorod, 308015, Russia \\ ${ }^{2}$ Vladimir State University, 87 Gor'kogo St, Vladimir, 600000, Russia
}

Published online: 15 February 2017

\begin{abstract}
Annotation: In this paper, methods of the implementing filtering are considered for recording of irregular disturbances of the geomagnetic field. The solution of problems of the processing of signals is considered for the system of the geodynamic control. They are based on the detection of the informative area of the registration in real time and the localization of the spatial location of the source of geomagnetic disturbances on the base of the regression analysis. It is noted that methods of simultaneous formation of borders of the bands of HPF and LPF necessary to use with the technical implementation of multi-band filtering. It allows to obtain the absolutely exact conjugation of frequency borders of neighboring subbands of filter, and also to reduce the number of tunable passive elements. Determined that in the technical implementation of the approach, obtaining an arbitrary phase-frequency characteristic that is not related to the amplitude-frequency response, and ensuring the stability of a multi-band filter of high order is important. Application of the regression analysis for selection and an assessment of parameters of distribution of geomagnetic pulsations at magnetotelluric sounding give the chance to consider features of use in system of monitoring of multirange filters and algorithms of spectral timing analysis.
\end{abstract}

Keywords: geomagnetic field, disturbance, registration, processing, signal, geodynamic, monitoring, magnetotelluric.

\footnotetext{
Author Correspondence, e-mail: gahov@bsu.edu.ru

doi: http://dx.doi.org/10.4314/jfas.v9i1s.745
} 


\section{INTRODUCTION}

The application of magnetotelluric sounding methods is consists in the measuring and the interpreting of recording signals of natural electromagnetic fields on the Earth's surface by systems of the geodynamic monitoring. Modern systems of the monitoring of the geomagnetic field of the Earth can to provide the reliable recording of signals of geomagnetic pulsations in the frequency range $0.001 \mathrm{~Hz}$ to $10 \mathrm{~Hz}$ [1-3]. Since the intensity of recording geomagnetic oscillations has the strongly marked frequency dependence. The necessity of measurements arises in several frequency ranges independently of each other [4]. This problem is solved by introducing into the measuring tract of geomagnetic measurement complexes the conjugate multiband filters and by the using of algorithms of the distributed processing on the network of monitoring stations synchronously in all ranges.

The processing of geomagnetic signals can be divide into stages during magnetotelluric sounding. They are determined by the system organization of monitoring processes of the geomagnetic field of the Earth's [5]. At the first stage, the registering of signals of geomagnetic pulsations necessary conduct in several frequencies bands synchronously at all monitoring stations. At the same time the current working frequency band necessary to form. In the second stage, a time interval is selected that contains the largest information in the selected frequency band with simultaneous spectral processing of the signal in real time. In the third stage, the estimate of the reliability of the information is carrying out on the base of algorithms of the localizing of the source of the geomagnetic disturbance. If necessary, the adjust of the choice of the frequency range is carrying out. At the same time, the reliability and the sufficient accuracy necessary to ensure when signals of geomagnetic pulsations are recorded and extracted for the implementation tasks of the geodynamic control.

\section{Fundamentals of the construction of conjugate filters}

Technically, the multi-band filtering can be implemented by using independent band-pass filters, that contain consistently included active filters of the upper (HPF) and lower (LPF) frequencies. The parallel inclusion of a certain number of filters allows the filtering of the signal in several frequency subbands at their outputs [6]. Weakness of this solution are the unclear coupling of frequencies borders of subbands and the large number of elements of active filters of hi- and low-frequencies, that necessary to tune. The first case forces to use overlap of the frequency borders because the loss of information is possible. Therefore, methods of simultaneous formation of borders of the bands of HPF and LPF necessary to use with the technical implementation of multi-band filtering. It allows to obtain the absolutely 
exact conjugation of frequency borders of neighboring subbands of filter, and also to reduce the number of tunable passive elements.

When cascade implementation of the HPF is used, the transmission function of the HPF can be written as:

$$
K_{B}(p)=\prod_{i=1}^{n} \frac{P_{i}(p)}{Q_{i}(p)},
$$

where $P_{i}(p), Q_{i}(p)$ - are polynomials of transmission functions of $i$-th cascade; $n$ - the number of cascades of HPF.

Then, we can to realize the LPF under certain conditions on base the k-th element of HPF with the transmission function:

$$
K(p)=1-K_{k}(p)=1-\frac{P_{k}(p)}{Q(p)},
$$

where $Q(p)=\prod_{i=1}^{n} Q_{i}(p)$.

Believing that the transmission function of the LPF (1) is conjugate with the transmission function of the HPF:

$$
K(p)=1 / Q(p),
$$

we define the required transmission function

$$
K_{k}(p)=\frac{Q(p)-1}{Q(p)} .
$$

The transsmission function (2) can to realize only for the certain structure of the HPF with the coefficient of the transmission of the k-th element of the filter

$$
K_{k}(p)=K_{B}(p) / K_{0 i}(p)
$$

where $K_{0 i}(p)$ - is the remaining part of the transmission of the tract of the HPF without $K_{k}(p)$.

For HPF of the second-order with transmission function

$$
K(p)=\frac{p^{2}}{1+p b_{1}+p^{2}}
$$

we can to use only one element of the HPH with a transmission function (3)

$$
K_{1}(p)=\left(\frac{p^{2}}{Q(p)}\right) /\left(\frac{P_{2}(p)}{Q_{2}(p)}\right),
$$


where $\frac{P_{2}(p)}{Q_{2}(p)}=\frac{p b_{2}}{1+p b_{2}}$ - is the transmission function of the second element with open feedback; $b_{1}, b_{2}$ - are coefficients of the polynomial of the transmission function and the second element, respectively.

Taking into account the above relations

$$
K_{1}(p)=\frac{p^{2}+p / b_{2}}{p^{2}+p b_{1}+1}
$$

with condition of the physical feasibility $b_{1} b_{2}=1$.

In the final analysis, filters of the measuring tract of the system of the monitoring of geomagnetic pulsations are realized on the base of some known structure (Sallen-Key, Kundert, Rauch et al., [7]). The choice of structure depends on the basic requirements for multi-band filters.

For the HPF of the Kundert structure (Fig. 1), the relations (4), (5) take the form for the transmission coefficients

$$
\begin{gathered}
K(p)=\frac{K_{0}-p^{2} \tau_{1} \tau_{2}}{1+p\left[\tau_{1}+\tau_{2}\left(1+R_{1} / R_{2}-K_{0}\right)+p^{2} \tau_{1} \tau_{2}\right]} \\
K_{1}(p)=\frac{p \tau_{1}\left(1+p \tau_{2}\right)}{Q(p)} \\
K_{2}(p)=\frac{1-p \tau_{2}\left(1+R_{1} / R_{2}-K_{0}\right)}{Q(p)}
\end{gathered}
$$

where $K_{0}=1+R_{0} / R, \tau_{1}=R_{1} C_{1}, \tau_{2}=R_{2} C_{2}$.

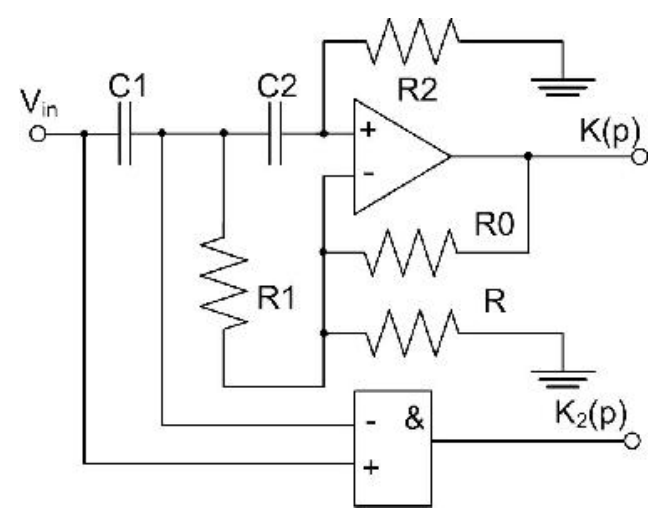

Fig.1. Conjugate filters on the Kundert structure

Normalized coefficients can be expressed in accordance with relations (6) 


$$
b_{1}=\frac{\tau_{1}+\tau_{2}\left(1-K_{0}\right)}{\sqrt{\tau_{1} \tau_{2}}}, \quad b_{2}=\frac{\tau_{2}}{\sqrt{\tau_{1} \tau_{2}}}
$$

The (7) substituting into (5), we determine that the relation is satisfied in the Kundert structure only in the case $K_{0}=1$. The ratio of the time constants corresponding to the implementation condition is

$$
\tau_{1} / \tau_{2}=b_{1}^{2}
$$

and does not limit the choice of the approximation of the amplitude-frequency characteristic.

For the HPF of the Sullen-Key structure (Fig. 2), the equations of base transmission coefficients (6) have the form

$$
\begin{gathered}
K(p)=\frac{K_{0} p^{2} \tau_{1} \tau_{2}}{1+p\left[\tau_{1}+\tau_{2}\left(1+K_{0}\right)+p^{2} \tau_{1} \tau_{2}\right]}, \\
K_{1}(p)=\frac{p \tau_{1}\left(1+p \tau_{2}\right)}{Q(p)}, \\
K_{2}(p)=\frac{1-p \tau_{2}\left(1-K_{0}\right)}{Q(p)} .
\end{gathered}
$$

The realization of the low-pass filter on the basis of the Sallen-Key HPF structure under the condition $K_{0}=1+R_{1} / R_{2}$ for the same ratio of time constants $\tau_{1} / \tau_{2}=b_{1}^{2}$ is performed. It can be seen from the relations (8).

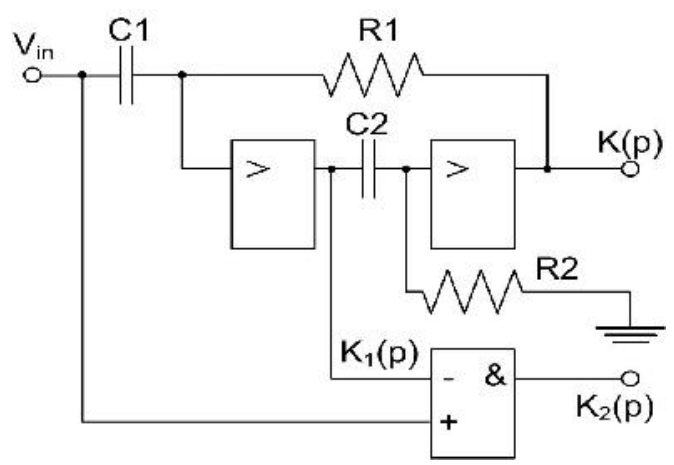

Fig.2. Conjugate filters on the Sallen-Key structure

The considered approach of constructing the conjugated HPFs and LPFs allows reducing the number of used highly stable passive elements. It's significantly increasing the metrological parameters of the system and providing a reliable conjugate between the frequency boundaries of the ranges of recording geomagnetic pulsations. The use of HPF with capacitive 
junctions as a base link ensures stability of operation and reduces the drift at the output of the filters.

Conjugate multi-band filters. Simplicity of conjugation of frequency boundaries of neighboring subbands and reduction of the number of tunable passive elements is provided in the technical implementation of the approach. In addition, obtaining an arbitrary phasefrequency characteristic that is not related to the amplitude-frequency response, and ensuring the stability of a multi-band filter of high order is important. The goal can be achieved by including combiners in a multi-band active filter, which contains a set of active multi-link high-pass filters connected in series (as the boundary frequencies increase). In this case, the number of adders is equal to the number of subbands in accordance with the considered principle of organizing conjugate filters [8]. In this case, the inputs of each adder to the input and output of the links of the corresponding active multi-link high-pass filter are connected. Such a connection eliminates the possibility of feedbacks through the adder between the links of the high-order active multi-tier filter. This ensures that the stability of this filter with the adders connecting is maintained. The characteristic equation of the transfer function of the adder together with the filter is determined by the characteristic equation of the same multilink filter. Since the transfer function of the adder is determined by independent summation coefficients, it allows obtaining an arbitrary phase-frequency characteristic of the filter at the output of the adder due to the realization of the non-minimal-phase transfer function

An example of a specific implementation of a single device range is the functional scheme of a three-link high-pass and adder filter, shown in Figure 3.

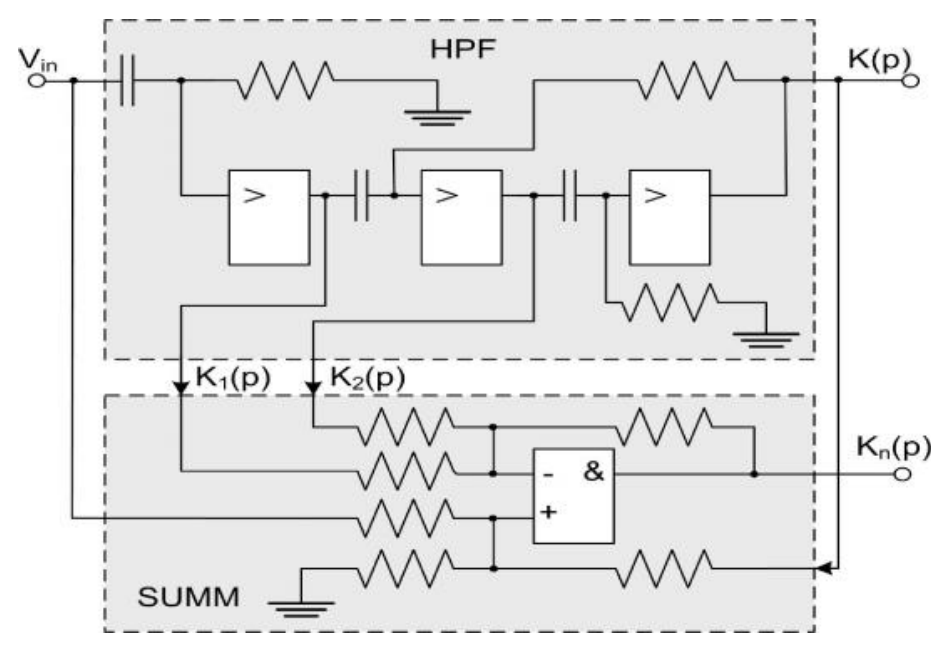

Fig.3. Functional scheme of the bandpass filter 
At the inputs of the adder, in accordance with the filter structure, transfer functions are realized:

$$
\begin{gathered}
K(p)=\frac{p a}{Q_{1}(p)}, \\
K_{1}(p)=\frac{K(p)\left(Q_{2}(p)-1\right)}{Q_{2}(p)}, \\
K_{2}(p)=\frac{K(p) p^{2}}{Q_{2}(p)},
\end{gathered}
$$

where $Q_{1}(p)=1+p a ; \quad Q_{2}(p)=1+p b_{1}+p^{2} ; a, b$ are coefficients of polynomials $Q_{1}, Q_{2}$

At the output of the adder, depending on the weight coefficients $k_{1}, k_{2}, k_{3}$, the transfer function of the low-pass filter is formed:

$$
K(p)=1+k_{1} K(p)+k_{2} K_{1}(p)+k_{3} K_{2}(p)=P(p) / Q(p) .
$$

In the particular case of the Chebyshev filter (with $P(p)=1$ ), the weight coefficients from the system of equations are determined:

$$
\begin{gathered}
(a+b)+k_{1} a=0, k_{1}=-(1+b / a) ; \\
(1+a b)+a b\left(k_{1}+k_{2}\right)=0, k_{2}=-\left(1-b^{2}\right) /(a b) ; \\
a+a\left(k_{1}+k_{2}+k_{3}\right)=0, \quad k_{3}=1 /(a b)>0 .
\end{gathered}
$$

The system of equations (10) can be continued for any number of links $P(p)$ of HPF and any value, including zeros in the right p-half-plane. The characteristic equation of the transmissions at the output of the HPF and at the output of the adder is determined by the same time constants of the HPF links. This simplifies conjugation of subbands at the cutoff frequency and makes it possible to reduce the number of tuned filter links. In addition, there are no feedback links between the HPF links through the adder, which ensures the stability of the HPF in any order. This allows us to use high order filters and makes it possible to obtain an arbitrary phase characteristic at the output of the adder by changing the weighting coefficients of the summation $k_{i}$.

Preliminary processing and selection of geomagnetic pulsations. When geomagnetic pulsations are used in the system of geodynamic monitoring, it is necessary to separate the individual spectral components of the signals of the geomagnetic field with certain properties. One of the methods of preliminary processing of geomagnetic signals is spectral-temporal 
analysis (STA), which allows identifing the main frequency components of the signal with minimal phase distortions on a distributed system of observations [9]. It consists of synchronous transmission of the input signal through a system of narrow-band filters and analysis of the amplitude values of the envelopes and phases at their output. In the real time automated measuring geophysical complex, traditional STA becomes unrealizable for a number of reasons and an algorithm based on the use of the complex demodulation method is used [10]. The main problem in its application is the matching of the necessary bandwidths of equivalent filters with a digital low-pass filter and the spectral composition of the analyzed wave packets.

If we assume that the signal of the analyzed wave packet is in the frequency range from $\omega_{1}$ to $\omega_{N}$, then for the implementation of the STA algorithm it is necessary covering this range with $N$ filters. To ensure the necessary accuracy of selection of geomagnetic pulsations, it is necessary that the amplitude-frequency characteristics of bandpass filters with bandwidth $2 \Delta \omega$ (for low-frequency filters in the complex demodulation procedure $\Delta \omega$, respectively) intersect at a level of $-3 \mathrm{~dB}$.

Including the overlapping parameter of the frequency range,

$$
\gamma=\left(\omega_{N} / \omega_{1}\right)^{\frac{1}{N-1}}
$$

we obtain the logarithmic scale of the central frequencies of the filters, the most common in known STA systems:

$$
\omega_{i+1}=\gamma \omega_{i}, \quad \omega_{i}=\gamma^{i-1} \omega_{1}
$$

and the bandwidth of the $i$-th filter will be equal to:

$$
\Delta \omega_{i}=(1-\gamma) \omega_{i} /(1+\gamma)
$$

The formulas (11), (12) allow setting the central frequencies and bandwidths of the filter system for the STA algorithm using the complex demodulation scheme. The accuracy of the algorithm and its time characteristics are determined by the applied digital low-pass filters, which provide the isolation of individual spectral components.

The digital recursive filters using in the STA algorithm and relating the input and output functions are described by a discrete-difference equation

$$
y[i]=\sum_{k=0}^{N} B_{k} x[i-k]-\sum_{m=1}^{M} A_{m} y[i-m] .
$$


In this equation, $N$ is the filter span (the number of input values used); $M$ is filter order (determined by the number of values of the preceding samples in the difference equation); $B_{k}, A_{m}$ are the coefficients of the filter.

The analysis of digital filters has shown that the digital filter of low frequencies with infinite impulse response (IIR) is optimal for the system of real-time magnetotelluric sounding, the realization of which does not require the accumulation of input data

$$
y[i]=B_{0} x[i]-\sum_{m=1}^{M} A_{m} y[i-m] .
$$

It has only the poles of the transfer function. One point at the output is calculated from one point entering the filter input.

The representation of the filter in the form (13) is its direct realization. It can also be represented as a cascade implementation by peer-to-peer filters and at each p-step of the filtering algorithm operation is performed:

$$
y_{p}[i]=b_{0} y_{p-1}[i]-a_{1 p} y_{p}[i-1]-a_{2 p} y_{p}[i-2] .
$$

Here $y_{0}[i]=x[i]$ is the input of the filter; $y_{R}[i]=y[i]$ is its output, and for each cascade $y_{p-1}[i]$ there is a cascade input and $y_{p}[i]$ - its output; $R$ is the number of cascades.

Accordingly, the transfer function of the $p$-th cascade is:

$$
H_{p}(j \Omega)=\frac{Y_{p}(j \Omega)}{X_{p}(j \Omega)}=\frac{b_{0}}{1+a_{1 p} \exp (-j \Omega T)+a_{2 p} \exp (-j 2 \Omega T)},
$$

where $T$ is the sampling period, and for all $R$-cascades

$$
H(j \Omega)=\frac{b_{0}^{R}}{\prod_{p=1}^{R}\left[1+a_{1 p} \exp (-j \Omega T)+a_{2 p} \exp (-j 2 \Omega T)\right]} .
$$

The coefficient $b_{0}$ in formula (14) is decisive in assessing the robustness of software implementation of real-time filters.

The calculation of the amplitude-frequency characteristic (AFC) and phase-frequency characteristic (PFC) of low-frequency filters of the 2nd and 6th orders shows that the damping of the filters for the $2 \mathrm{nd}$ order is $11 \mathrm{~dB} /$ octave outside the passband $(0 \div-3 \mathrm{~dB})$, for the 4 th order - $22 \mathrm{~dB} /$ octave, For the 6 th $-34 \mathrm{~dB} /$ octave, and for the classical filter - $16 \mathrm{~dB} /$ octave. As can be seen from the above calculations of the AFC and PFC, Butterworth filters can be used in the complex demodulation algorithm in a manner similar to the filters used in the classical STA algorithm. 
The regression analysis algorithm of spectral-time analysis data. Definition of location of a source of geomagnetic pulsations and characteristics of their spatial distribution allows constructing exact model of a source of the field in a point of magnetotelluric sounding. It allows estimating at a stage of processing geodynamic reliability of the received results on the basis of statistical estimates. The main characteristics to be evaluated are the azimuth of the propagation direction, the phase velocity and the spatial damping of the wave [11]. It is obvious that heterogeneity of the considered field assumes spectral and spatial heterogeneity of the geomagnetic pulsations sources. Accordingly, it is possible to determine its characteristics only for individual components of the spectral expansion of the field, which are obtained as a result of processing the signals of the geomagnetic field on the network of stations using the algorithm of spectral-temporal analysis (STA).

In an algorithm of the regression analysis it is necessary to consider conditions of registration of signals of geomagnetic pulsations. First of all it is necessary to consider influence of the used multirange filters in a measuring path, and also parameters of STA filters [12]. Distinctive feature of the considered algorithm is statistical definition of phase amendments with correction of phase distortions which are brought by the applied signal filtration.

The source of the geomagnetic disturbance can be located at an arbitrary direction angle to the geomagnetic signal recording systems on the network of observation stations and taking into account the elliptical polarization of the spatial harmonics of the field of geomagnetic pulsations. In this case, it is necessary to use data in the regression analysis algorithm that are invariant to the spatial orientation of signal recording systems [13]. These include the values of the large axes of the ellipses of polarization $A_{0 i}\left(\omega_{j}\right)$ and phase $F_{0 i}\left(\omega_{j}\right)$, which correspond to the coincidence of the vector of the spectral component $\omega_{j}$ STAN with the major axis of the polarization ellipse at the i-th observation station. They are defined on the basis of amplitude data of spectral timing analysis $H_{x i}\left(\omega_{j}\right)$ and $H_{y i}\left(\omega_{j}\right)$, and also on the basis of phase data $F_{x i}\left(\omega_{j}\right)$ and $F_{y i}\left(\omega_{j}\right)$ for the corresponding frequency $\omega_{j}$ in accordance with the following relationships:

$$
\begin{aligned}
& A_{0 i}^{2}=\frac{1}{2}\left[H_{x i}^{2}+H_{y i}^{2}+\sqrt{\left(H_{x i}^{2}+H_{y i}^{2}\right)^{2}-4 H_{x i}^{2} H_{y i}^{2} \sin \left(F_{y i}-F_{x i}\right)}\right] \\
& \operatorname{tg} F_{0 i}=\frac{H_{x i}^{2} \sin \left(F_{x i}\right) \cos \left(F_{x i}\right)+H_{y i}^{2} \sin \left(F_{y i}\right) \cos \left(F_{y i}\right)}{A_{0 i}^{2}-\left[H_{x i}^{2} \sin ^{2}\left(F_{x i}\right)+H_{y i}^{2} \sin ^{2}\left(F_{y i}\right)\right]} .
\end{aligned}
$$


In this case, the estimate of the epicenter of the geomagnetic disturbance can be carried out according to (15) on the basis of a linear regression model:

$$
\begin{gathered}
F_{0 i}\left(\omega_{j}\right)=\varphi_{0 i}\left(\omega_{j}\right)-b\left(\omega_{j}\right) r_{i}-L_{\varphi}[i, j], \\
\ln \left[A_{0 i}\left(\omega_{j}\right)\right]=\ln \left[\hat{A}_{0}\left(\omega_{j}\right)\right]-\alpha\left(\omega_{j}\right) \ln r_{i} .
\end{gathered}
$$

In the above expression (16), $\alpha\left(\omega_{j}\right)$ and $b\left(\omega_{j}\right)$ are regression coefficients that determine the geomagnetic disturbance propagation parameters, $r_{i}$ - distances from the observation stations to the assumed epicenter, $\varphi_{0 i}\left(\omega_{j}\right)$ and $\ln \left[\hat{A}_{0}\left(\omega_{j}\right)\right]$ are the given values of phases and a logarithm of amplitude of a source in an epicenter zone.

Elements of a matrix of phase correction $L_{\varphi}[i, j]$ are defined by the equation

$$
L_{\varphi}[i, j]=2 \pi n[i, j]+\Delta \varphi[i, j]
$$

where $\Delta \varphi[i, j]$ - correction of phase distortions introduced by multi-band filters; $n[i, j]$ phase additives that take into account the wave propagation of the fields of geomagnetic pulsations.

In accordance with the regression analysis algorithm [14] and relations (16), (17), propagation parameters can be determined by formulas

$$
\begin{gathered}
\alpha\left(\omega_{j}\right)=\frac{\sum_{i=1}\left[\overline{\ln \left(A_{0 i}\right) r}-\overline{\ln \left(A_{0 i}\right)} \bar{r}\right] \omega_{j} \delta_{i j}}{\sum_{i=1}\left[\overline{\ln \left(r^{2}\right)}-\ln (\bar{r})^{2}\right] \omega_{j} \delta_{i j}}, \\
b\left(\omega_{j}\right)=\omega_{j}^{\beta} \frac{\sum_{i=1}\left[\overline{F_{0 i} r}-\overline{F_{0 i}} \bar{r}\right] \omega_{j} \delta_{i j}}{\sum_{i=1}\left[\overline{r^{2}}-\bar{r}^{2}\right] \omega_{j} \delta_{i j}},
\end{gathered}
$$

where $\beta$ - Dispersion coefficient reflecting the features of the propagation of pulsations type geomagnetic; $\delta_{i j}$ - weighting factor that takes into account the bandwidth of the filters of the spectral-time analysis algorithm at the filter setting frequency $\omega_{j}$ and the noise level at the $i$-th monitoring station

At the same time accuracy of localization of epicenter of a source of a geomagnetic disturbance can be estimated by means of the generalized values of coefficients of correlation according to amplitude $R_{a}$ and phase $R_{\varphi}$ data of STA. 
The Table 1 gives an estimate of the propagation parameters of geomagnetic pulsations of the Pi-2 type. These pulsations are registered on the network of geophysical stations for the average periods of wave packets $T_{\text {avg }}$. The evaluation was carried out in accordance with the proposed algorithms.

The last column gives the values of the phase velocities calculated by formula

$$
V_{f}=360 / b\left(T_{\text {avg }}\right) T_{\text {avg }} .
$$

Table 1. Estimation of the distribution parameters of geomagnetic pulsations

\begin{tabular}{|c|c|c|c|c|c|c|c|}
\hline $\mathrm{N}$ & Time on UT & $T_{\text {avg }}$ & $\alpha\left(T_{\text {avg }}\right)$ & $b\left(T_{\text {avg }}\right)$ & $R_{\varphi}$ & $R_{a}$ & $V_{\varphi}$ \\
\hline 1 & $1747: 29.6$ & 30.6 & 1.443 & 0.749 & 0.99988 & 0.980 & 15.7 \\
\hline 2 & $1749: 31.2$ & 30.6 & 1.456 & 0.912 & 0.99688 & 0.962 & 12.9 \\
\hline 3 & $1753: 28.0$ & 49.4 & 1.679 & 0.681 & 0.99959 & 0.989 & 10.7 \\
\hline 4 & $1753: 15.2$ & 28.4 & 1.415 & 0.939 & 0.99609 & 0.979 & 13.5 \\
\hline 5 & $1756: 01.6$ & 37.5 & 1.502 & 0.681 & 0.99277 & 0.956 & 14.1 \\
\hline 6 & $1759: 58.4$ & 25.0 & 1.408 & 1.091 & 0.99979 & 0.939 & 13.2 \\
\hline 7 & $1805: 18.4$ & 53.0 & 1.790 & 0.581 & 0.99843 & 0.990 & 11.7 \\
\hline 8 & $1831: 42.4$ & 33.4 & 1.486 & 0.829 & 0.99641 & 0.932 & 13.0 \\
\hline 9 & $1836: 24.0$ & 45.0 & 1.544 & 0.741 & 0.99936 & 0.960 & 10.8 \\
\hline 10 & $1840: 01.6$ & 35.6 & 1.496 & 0.760 & 0.99663 & 0.996 & 13.3 \\
\hline
\end{tabular}

Apparently from the table, the values of coefficients of correlation determined by the offered algorithm have high values. It testifies to accuracy of work of the considered algorithm of regression processing for localization of epicenters of geomagnetic pulsations and definition of characteristics of their horizontal distribution. The obtained values of the phase velocities of pulsations of the Pi-2 type agree well with the theoretically estimated ones and decrease with increasing period.

\section{CONCLUSION}

Investigation of the geomagnetic field of the Earth allows not only to better understand the laws of its change and its connection with processes of both planetary and other origin, but also, based on the results of this study, to carry out monitoring and preventive measures to protect industrial facilities and the biosphere from the origin of causes causing technogenic catastrophe.

The inhomogeneity of the geomagnetic field in question implies the spectral-spatial inhomogeneity of the source of geomagnetic signals. In this case, the processing should provide separation of signals of unitary irregular geomagnetic perturbations and the possibility of spatial point approximation of the source with a limited statistical accuracy. The problem is solved by realizing a magnetotelluric sounding with the use of the filter 
conjugation technique and the data processing algorithms of STA, which was described in this article.

\section{SUMMARY}

As a result of the research it was established:

- The use of multi-band active filters as a high-frequency baseband built on the basis of the standard Kundert and Sallen-Key structures makes it possible to ensure the accuracy of conjugation of frequency boundaries, as well as to simultaneously monitor geomagnetic disturbances in several frequency ranges.

- The quality and reliability of selection of geomagnetic pulsations in the system is determined by the methods of preliminary processing of the recorded data, built on the algorithms of time-spectral analysis in real time.

- Application of the regression analysis for selection and an assessment of parameters of distribution of geomagnetic pulsations at magnetotelluric sounding gives the chance to consider features of use in system of monitoring of multirange filters and algorithms of spectral timing analysis.

\section{REFERENCES}

1. Tran Trong Lap, Chuandong Xue, Aiying Wei, Lv Liu, Wenyao Li, Qiquan Hu, Jingjie Li, Dafeng Luo, Shaoyong Zhu, Tiangui Zhang , 2014. Application of Audio-Magnetotelluric Method for Exploration the Concealed Ore-Bodies in Yuele Lead-Zinc Ore Feild, Daguan County, NE Yunnan Province, China, Journal of Geoscience and Environment Protection, 2: 35-45

2. Vartanyan, G. S., 2010. Regional geodynamic monitoring system for ensuring safety in geological and exploratory production of oil and gas, Izvestiya, Atmospheric and Oceanic Physics, 46(8): 952-964

3. Jeffrey J. Love, 2008. Magnetic monitoring of Earth and space, Physics Today, February, pp.31-37.

4. Bath, B.M., 1982. Spectral Analysis in Geophysics. 1st Edition, Elsevier Science, 1st January, $580 \mathrm{p}$.

5. Dmitriev, V.I., Berdichevsky, M.N., 1979. The Fundamental Model of Magnetotelluric Sounding, Proceedings of the IEEE, 67(7):1034-1044.

6. Lacanette, K., 1991. A Basic Introduction to FiltersĐActive, Passive, and SwitchedCapacitor, National Semiconductor, Application Note 779, April. 
7. Walter E. Holmes, Harvey W. Heinlein, 1974. Active filters for integrated circuits : fundamentals and design methods, R. Oldenbourg: VerlagSpringer-Verlag, New York.

8. Kuzichkin, O.R., Kuligin, M.N., Tsaplev, A.V. et al., 2006. Multi-band Active Filter. Patent of the Russian Federation № 55232, Bulletin №21 (in Russian).

9. Eidsvik, J., Mukerji, T., Bhattacharjya, D., Watson, T.J., Dutta, G. ,2015. Value of Information Analysis of Geophysical Data for Spatial Decision Situations. SEG Technical Program Expanded Abstracts: 2858-2862.

10. Kuzichkin, O.R., 2006. Spectral-time analysis in real time. Collection "Modern problems of radio electronics", Issue 1, Rostov-on-Don, Rostov State Pedagogical University (in Russian).

11. Nan-Wei Chen, Jann-Yenq Liu, 2008. Global ultra-Low-Frequency Geomagnetic Pulsations Associated with the March 24, 1991 Geomagnetic Storm, Terr. Atmos. Ocean. Sci., 19 (3): 291-308.

12. Bleier, T., Dunson, C., Maniscalco, M., Bryant, N., Bambery, R., Freund, F., 2009. Investigation of ULF magnetic pulsations, air conductivity changes, and infra red signatures associated with the 30 October Alum Rock M5.4 earthquake, Nat. Hazards Earth Syst. Sci., 9: $585-603$.

13. Sharapov, R., Kuzichkin, O.,2013. The polarizing characteristics of electrolocation signals and then analysis in geomonitoring system. Geoconference on science and technologies in geology, exploration and mining, SGEM, Vol II: 913-918.

14. Bykov, A.A., Kuzichkin, O.R., 2014. Regression Prediction Algorithm Of Suffusion Processes Development During Geoelectric Monitoring, Advances in Environmental Biology, 8(5): 1404-1408.

15. Ivashuk, O.A., Konstantinov, I.S., Shcherbinina, N.V., Kvanin, D.A., Gakhov, R.P., 2015. Automated Management of Biotechnosphere of Local Urban Areas. International Business Management, 9 (7): 1598-1603.

16. Ivashuk, O.A., Shcherbinina, N.V., Lazarev, S.A., Ivashchuk O.D., 2015. Increasing Efficiency Territory Technospheric Safety Management Based on Improving of Earth's Surface Satellite Images. International Journal of Soft Computing, 10(6): 420-426.

17. Chernomorets, A.A., Petina ,M.A., Kovalenko, A.N., Zaitseva, N.O. 2017.Groundwaters graphic-analytical model of dynamics of distribution. Belgorod State University Scientific Bulletin, Ser.Economics, Information technologies, № 2 (251), Issue 41: 75-80 (In Russian). 


\section{How to cite this article:}

Kuzichkin O R, Grecheneva A V, Gakhov R P, Dorofeev N V, Bykov A A, Gakhov B R. The registration and the processing of signals of geomagnetic pulsations in the system of the geodynamic monitoring. J. Fundam. Appl. Sci., 2017, 9(1S), 914-928. 